\title{
Drenaje anómalo total de venas pulmonares variante supracardiaca en una mujer adulta
}

\section{Supracardiac total anomalous pulmonary venous connection in an adult woman}

\author{
Javier López ${ }^{1 *}$, Irene Torres ${ }^{1}$, Jennifer Pacheco², Richard Quizhpe ${ }^{3}$ y Juan Flores ${ }^{1}$ \\ ${ }^{1}$ Servicio de Cirugía Cardiotorácica; ${ }^{2}$ Servicio de Hemodinámica; ${ }^{3}$ Servicio de Cardiología. Hospital de Especialidades José Carrasco Arteaga, \\ Cuenca, Azuay, Ecuador
}

La conexión anómala total de venas pulmonares (CATVP) es una cardiopatía congénita en la que la circulación venosa pulmonar drena al sistema venoso sistémico ${ }^{1}$. La existencia de una comunicación interauricular es imprescindible para la supervivencia². Según la clasificación de Darling modificada, puede ser supradiafragmática, infradiafragmática o mixta, dividiéndose la primera en supracardiaca, cardiaca e infracardiaca ${ }^{3}$.

Su incidencia mundial es de 2/100.000 nacimientos ${ }^{4}$ y representa el $1-3 \%$ de todas las cardiopatías congénitas $^{1}$; la más frecuente es la supracardiaca ${ }^{4}$. Se produce durante el desarrollo intrauterino y se debe a la persistencia funcional del sistema venoso primitivo ${ }^{5}$.

El diagnóstico se realiza mediante ecocardiografía. La tomografía computarizada o el cateterismo son útiles si existen dudas o coexistencia de anomalías asociadas $^{2}$. Desde la obtención del diagnóstico definitivo existe ya una indicación de corrección quirúrgica. La evolución natural en corazones biventriculares sin reparación quirúrgica indica una mortalidad del $80 \%$ dentro del primer año de vida ${ }^{1}$.

Las principales causas de muerte hospitalaria son el edema pulmonar agudo y las crisis de hipertensión pulmonar. Como complicaciones se mencionan también arritmias, bajo gasto cardiaco y obstrucción de venas pulmonares residual ${ }^{1}$. Son factores de riesgo la variante infracardiaca, la presencia de obstrucción, el estado crítico preoperatorio, la hipoplasia de venas pulmonares y la presencia de otra malformación congénita mayor coexistente 1 .

Presentamos el caso de una paciente de 36 años que cursó dos embarazos con partos eutócicos no complicados, posterior a los cuales presenta dolor de pecho y disnea de grandes esfuerzos, misma que progresa hasta pequeños esfuerzos al momento del ingreso y se le suma cianosis. En el corazón se ausculta un soplo holosistólico en foco pulmonar y tricuspídeo. En la radiografía de tórax se observa el signo del muñeco de nieve y el electrocardiograma muestra un patrón de bloqueo de rama derecha.

En las pruebas de imagen se evidencia una dilatación importante de las cavidades derechas, fracción de eyección del ventrículo izquierdo del $75 \%$, insuficiencia tricuspídea moderada, comunicación interauricular y CATVP variante supracardiaca. El cateterismo realizado previo al ingreso reporta hipertensión pulmonar moderada (presión sistólica de la arteria pulmonar [PSAP]: $45 \mathrm{mmHg}$ ).

Se realiza corrección quirúrgica total por abordaje posterior con un tiempo de circulación extracorpórea de 1 hora y 48 minutos, y un tiempo de pinzamiento de 1 hora y 30 minutos. Al realizar la esternotomía

\footnotetext{
Correspondencia:

Fecha de recepción: 04-03-2020

Disponible en internet: 03-11-2020

*Javier López

Fecha de aceptación: 02-07-2020

Arch Cardiol Mex. 2021;91(2):264-266

DOI: 10.24875/ACM.20000116

www.archivoscardiologia.com

E-mail: javieelopezz@gmail.com

www.archivoscardiologia.com

CC BY-NC-ND (http://creativecommons.org/licenses/by-nc-nd/4.0/).
} 


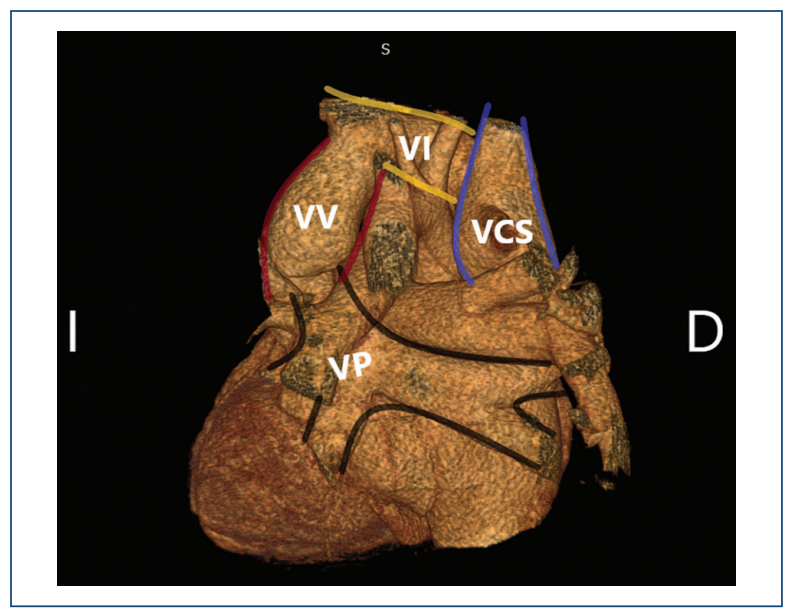

Figura 1. Angiotomografía, reconstrucción en tres dimensiones. Vista posterior. Se observa la conexión anómala total de venas pulmonares (VP) supracardiaca con colector que drena hacia una vena vertical (VV) que a su vez drena hacia la vena innominada (VI) y hacia la vena cava superior (VCS).

media longitudinal se observan las venas innominada y vertical muy dilatadas (los estudios de imagen previos indicaron la confluencia de las cuatro venas pulmonares hacia un colector retropericárdico que drenaba hacia la vena innominada a través de una vena vertical) (Fig.1). Se realiza una incisión longitudinal en el pericardio para rodear con una ligadura de seda la vena vertical y disecar totalmente el colector posterior. Una vez iniciada la circulación extracorpórea se procede a ligar la vena vertical. En paro electromecánico y temperatura de $28^{\circ} \mathrm{C}$ se inciden transversalmente el colector y el atrio izquierdo en su pared posterior hasta la orejuela izquierda, realizando una anastomosis laterolateral entre estas dos estructuras sin ocasionar tensión en los tejidos y evitando estenosis residuales en la anastomosis (Fig. 2). Se eligió este abordaje ya que es seguro, ofrece una exposición efectiva y no aumenta la incidencia de arritmias auriculares al no requerir más incisiones en las aurículas ${ }^{6}$. Mediante auriculotomía derecha se realiza el cierre de la comunicación interauricular con parche de pericardio bovino fenestrado.

La paciente permaneció 3 días en la unidad de cuidados intensivos, donde fue extubada en su primer día y permaneció con apoyo inotrópico con milrinona y norepinefrina durante 24 horas. Posteriormente tuvo una buena evolución, con ecocardiograma de control que reporta PSAP de $59 \mathrm{mmHg}$, septos íntegros y a nivel posterior de la aurícula izquierda flujo único de

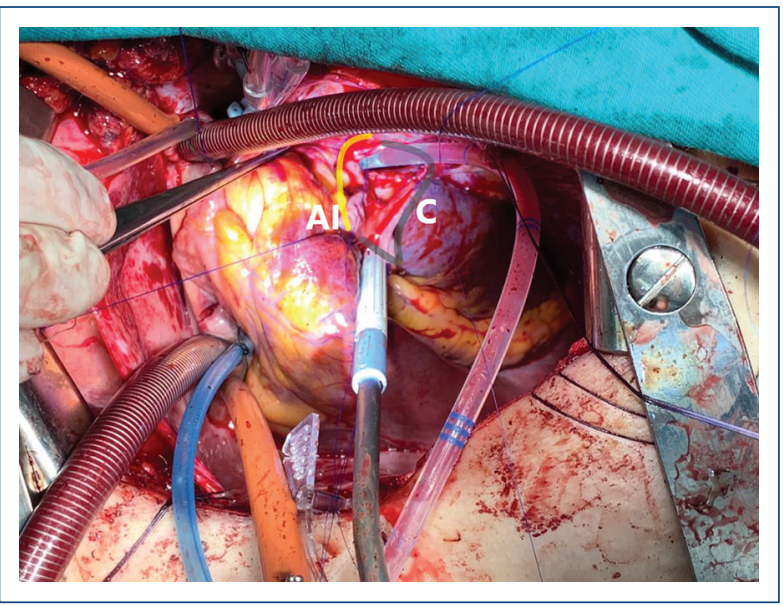

Figura 2. Anastomosis del colector hacia la aurícula izquierda. En bomba extracorpórea con corazón detenido y aorta pinzada, mediante abordaje posterior con luxación del corazón hacia el cirujano se realiza anastomosis del colector (C) hacia la pared posterior de la aurícula izquierda (AI).

colector de drenaje venoso. Recibe el alta médica 11 días tras el procedimiento.

El embarazo es un estado de alta demanda hemodinámica. El gasto cardiaco se eleva hasta un 100\% durante el tercer trimestre ${ }^{5}$, por lo que es común que durante la gestación las mujeres debuten con síntomas asociados a malformaciones congénitas de otra manera silentes. La CATVP asociada al embarazo es rara e incluso se considera una indicación de terminación del embarazo ${ }^{7}$. Llama la atención que esta paciente haya tenido dos embarazos y partos eutócicos durante los cuales permaneció asintomática y sin diagnóstico.

Esta evolución inusualmente tardía es atribuible a la afortunada presencia de algunos factores protectores conocidos, como tratarse de una variante supracardiaca, la ausencia de otras malformaciones concomitantes que compliquen el cuadro, una comunicación interauricular amplia que permitía el paso irrestricto de sangre sumado a una insuficiencia tricuspídea que a su vez aumenta este flujo hacia la aurícula izquierda, y la ausencia de obstrucción venosa en el trayecto hacia la aurícula derecha ${ }^{5}$.

Una vez realizado el diagnóstico se debe hacer una minuciosa valoración anatómica y clínica de cada caso para definir la conducta. Toma relevancia el cateterismo derecho de las presiones pulmonares, ya que así se pueden detectar etapas tardías de hipertensión pulmonar, complicación muy frecuente al ser parte de la evolución natural de la enfermedad, que podría incluso contraindicar la resolución quirúrgica ${ }^{8}$. Los estudios de imagen de 
nuestra paciente se realizaron a lo largo de varios meses, ya que reside en otra provincia y tenía dificultad de acceso. El cateterismo realizado inicialmente mostró una hipertensión pulmonar moderada, con lo que se decidió que era plausible aún la resolución quirúrgica.

Este procedimiento es poco común en adultos, por lo que su morbimortalidad perioperatoria no ha sido bien estudiada en este tipo de pacientes. Existen series de casos con pequeños grupos que reportan buena sobrevida ${ }^{6}$, mencionando como complicaciones distrés respiratorio, crisis de hipertensión pulmonar y bajo gasto cardiaco favorecidos por el hiperflujo pulmonar preexistente. Además, como complicación estructural más importante se debe tener muy en cuenta la estenosis residual en la anastomosis de las venas pulmonares hacia la aurícula izquierda, ya que se puede presentar hasta en el $19 \%$ de los casos 9 .

Se decidió desde un inicio colocar un parche fenestrado para prevenir complicaciones por hipertensión pulmonar grave en el posquirúrgico, pues a pesar de presentar hipertensión pulmonar moderada en el cateterismo inicial tuvo en el último mes deterioro de su clase funcional y se sumó cianosis a su sintomatología; estas son manifestaciones clínicas que indican un aumento de la hipertensión pulmonar. Además, pueden existir variaciones de la presión pulmonar secundarias a la altura; existen casos reportados en los que residentes en grandes alturas pueden presentar importantes incrementos de la presión pulmonar al cambiar de localidad ${ }^{10}$.

La evolución del cuadro fue excelente; el ecocardiograma posquirúrgico de control indica tabique interauricular sin flujos residuales, hipertensión pulmonar grave y adecuado flujo a nivel de la anastomosis de venas pulmonares sin estenosis.

Este caso es de relevancia ya que se trata de una paciente con una evolución peculiar de una CATVP. Llama la atención que, aunque se sabe que la presión arterial pulmonar de los habitantes de altura es mayor, no haya desarrollado hipertensión pulmonar grave a pesar de vivir en una ciudad situada a 2500 metros sobre el nivel del mar. De igual manera extraña su antecedente de edad adulta con varias gestaciones durante las cuales permaneció completamente asintomática a pesar de la aumentada demanda hemodinámica que esto representa.

Debido al riesgo de complicaciones con fallo derecho e hipertensión pulmonar, se decidió emplear un parche fenestrado para el cierre de la comunicación interauricular. El procedimiento se llevó a cabo por abordaje posterior con una adecuada visualización de las estructuras. Este es el primer caso de corrección total de una CATVP en Ecuador.

Nuestra paciente presentó una excelente evolución, con adecuada función hemodinámica y significativa mejoría en cuanto a sintomatología, por lo que tiene un buen pronóstico a largo plazo.

\section{Financiamiento}

Ninguno.

\section{Conflicto de intereses}

Los autores declaran no tener ningún conflicto de intereses.

\section{Responsabilidades éticas}

Protección de personas y animales. Los autores declaran que para esta investigación no se han realizado experimentos en seres humanos ni en animales.

Confidencialidad de los datos. Los autores declaran que han seguido los protocolos de su centro de trabajo sobre la publicación de datos de pacientes.

Derecho a la privacidad y consentimiento informado. Los autores han obtenido el consentimiento informado de los pacientes y/o sujetos referidos en el artículo. Este documento obra en poder del autor de correspondencia.

\section{Bibliografía}

1. Rivera Ladino KM, Naranjo Ugalde AM, Selman-Housein Sosa E, Frias F. Drenaje anómalo total de venas pulmonares, veinticuatro años de tratamiento quirúrgico. Cardiocentro William Soler. Rev Cubana Cardiol Cir Cardiova. 2013;19:21-4.

2. Aroca A, Polo L, Bret M, López-Ortego P, González A, Villagrá F. Drenaje venoso pulmonar anómalo total. Técnicas y resultados. Cir Cardiov. 2014;21:90-6.

3. Muñoz Castellanos L, Kuri Nivon M, Sánchez CA. Estudio morfopatológico de la conexión anómala total de venas pulmonares. Rev Arch Cardiol México. 2007;77:265-74.

4. Farfán E, Echeverría M, Salgado G, Navarro S, Inostroza A, Inzunza O. Conexión venosa pulmonar anómala. Reporte de un caso. Int J Morphol. 2018;36:1197-201.

5. Talib N, Sayuti K, Abdullah MS, Mohd Zain MR. Beating the odds: a rare case of supracardiac total anomalous pulmonary venous return (TAPVR) in an adult patient. BMJ Case Rep. 2018;2018:bcr2017221074.

6. Talwar S, Choudhary SK, Reddy S, Saxena A, Kothari SS, Juneja R, et al. Total anomalous pulmonary venous drainage beyond childhood. Interact Cardiovasc Thorac Surg. 2008;7:1058-61.

7. Bangal VB, Kanika G, Gavhane SP, Gangapurwala S. Rare case of pregnancy with total anomalous pulmonary venous connection (TAPVC). Int J Biomed Res. 2014:5:143-4.

8. Atik FA, Jaramillo M, Afiune JY, Caneo LF. Complete repair of total anomalous pulmonary venous connection in adult patient. Braz $\mathrm{J}$ Cardiovasc Surg. 2009:24:81-3.

9. Wu FM, Emani SM, Landzberg MJ, Valente AM. Rare case of undiagnosed supracardiac total anomalous pulmonary venous return in an adult. Circulation. 2014;130:1205-7.

10. Spielvogel H. Presión arterial pulmonar y altura. Cuad Hosp Clínicas. 2007;52:86-92. 